\title{
QoS Aware Logical Channel Prioritization under Burst Resource Allocation for Uplink in LTE
}

\author{
Mannu Kumar \\ Department of CSE, The NorthCap University, Gurgaon, India \\ E-mail: manu.itsolution@gmail.com \\ Neeti Kashyap \\ Department of CSE, The NorthCap University, Gurgaon, India \\ E-mail: neetikashyap@ncuindia.edu
}

Received: 26 January 2017; Accepted: 09 May 2017; Published: 08 August 2017

\begin{abstract}
Long Term Evolution (LTE) is the latest 3GPP ( $3^{\text {rd }}$ Generation Partnership Project) standard of the Mobile communication system. LTE has been proposed to achieve higher data throughput, lower latency and better quality of service (QoS). In LTE network (n/w), the resource (resource referred to frequency and time domain on the air interface) sharing is one of the major challenging issues and it is one of the key functions to achieve the desired QoS for the different configured data stream. In this context of QoS for LTE n/w, Multiplexing \& Logical Channel Prioritization (LCP) is referred. In this paper, we present a brief survey on LCP techniques for the uplink (UL) direction. UL direction referred mobile to $\mathrm{n} / \mathrm{w}$ communication. A strategy has been proposed for LCP to achieve QoS under burst resource allocation environment. Proposed approach considers the priority of each logical channel configured by evolved NodeB (eNB) during radio bearer (RB) setup/re-configuration.
\end{abstract}

Index Terms-LTE (Long Term Evolution), LCP (Logical Channel Prioritization), RB (Radio Bearer), eNB (Evolved NodeB), QoS (Quality of Service), MAC (Medium Access Control).

\section{INTRODUCTION}

The $4^{\text {th }}$ generation of mobile communication standard is known as Long Term Evolution (LTE). It is currently being deployed throughout the world. It is the latest deployed mobile communication technology which is successor of $3 \mathrm{G}$ technology and has an impressive impact on the world. The collective number of cellular subscriber is leading towards the world population count which is $\sim 7$ billion [1].

LTE first specification was published under 3GPP Release 8 [2]. The technology used in mobile

Communication has been growing very fast in the last few years and its development is still continuous. Currently, we can see the deployment of LTE $n / w$ all over the world. At the same time new technology is also being researched with the aim of better performance in term of data throughput, latency and QoS for the $5^{\text {th }}$ generation mobile communication [3].

LTE $\mathrm{n} / \mathrm{w}$ has been optimized for higher data throughput. The maximum data rate is up to $300 \mathrm{Mbps}$ for downlink and for uplink, it is up to 75Mbps [4] in Rel-8 standard, LTE-A (Advance) supports even higher speed. LTE is differing with its earlier communication standard at a large extent. The main features of LTE standard can be summarized as follows:

- Orthogonal Frequency Division Multiple Access (OFDMA) and Single Carrier Frequency Division Multiple Access (SC-FDMA) are used in DL \& UL direction respectively. These techniques allow the sharing of radio resources at the air interface (it is the interface between UE and eNB known as Uu interface).

- Transmission Time Interval (TTI) is basically the minimum time period for scheduling. At maximum, a UE can be scheduled in every TTI; duration of a TTI is 1 millisecond (ms). Thus LTE system has fast adaptability with the change of radio conditions.

- The core $\mathrm{n} / \mathrm{w}$ architecture has been simplified to the earlier standards. LTE $\mathrm{n} / \mathrm{w}$ is completely Internet Protocol (IP) based, means all interfaces between the different $n / w$ nodes are IP based except the air interface.

- LTE system provides the better voice quality with Voice over LTE (VoLTE) feature than the earlier standards. Higher codec rate during VoLTE call makes the better voice quality for end users.

\section{NETWORK ARCHITECTURE}

In this section, we will present the main $\mathrm{n} / \mathrm{w}$ elements of LTE $n / w$ architecture. Evolution of LTE air interface is referred to as Evolved Universal Terrestrial Radio Access Network (E-UTRAN) and evolution of core $\mathrm{n} / \mathrm{w}$ is referred to as Evolved Packet Core (EPC). The combined evolution of E-UTARN and EPC together is called 
Evolved Packet System (EPS) as shown in Fig. 1.

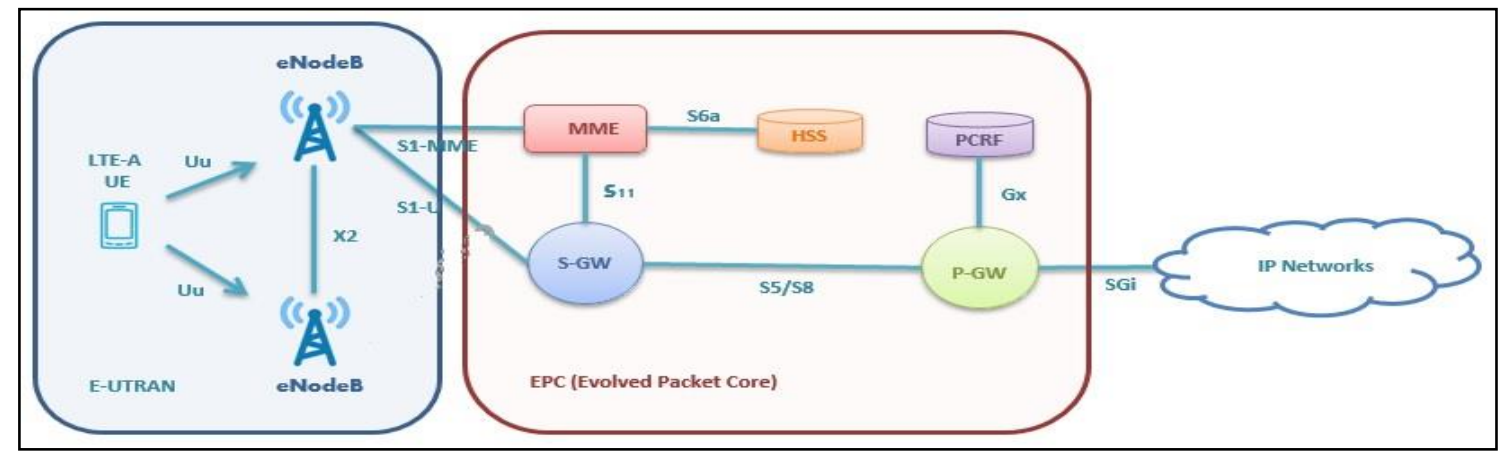

Fig.1. LTE Architecture

Fig. 2 shows the more generalize view of E-UTRAN part of LTE architecture. Communication of E-UTRAN with EPC is either signaling (control plane) or traffic (data plane) as shown in Fig. 2 [5].

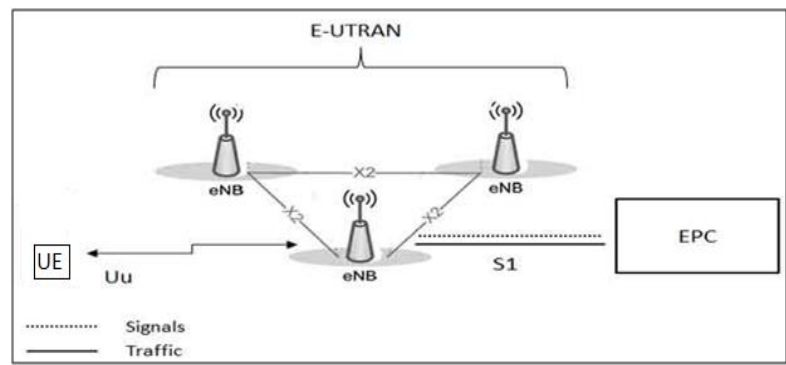

Fig.2. E-UTRAN

By referring the Fig. 1 and Fig. 2, the main component of LTE architecture or EPS architecture as follows:

- User Equipment (UE): It is basically a device used by end user for communication. Typically, it is smart phone, data card or hand held communicative device.

- E-UTRAN NodeB (eNB): It is the main controller for all air interface related functions. It resides between UE and the core n/w (EPC) and acts like layer 2 . The eNB is responsible for radio resource management i.e. resource allocation, traffic scheduling based on the QoS. It also plays a major role in mobility control of the UE in both idle and connected mode [6].

- Mobility Management Entity (MME): It is the main component in EPC. It works only in signaling plane. The main function of MME is authentication, security and mobility management [6].

- Service Gateway (S-GW): S-GW resides between the eNB \& P-GW thus it conveys the traffic between eNB and P-GW. It is used to initiate the paging for the incoming traffic at the UE [6].
- PDN Gateway (P-GW): It acts like a gateway between the LTE $\mathrm{n} / \mathrm{w}$ (EPS) and the external network. It allocates the IP address to the UE during the PDN connection procedure and UE uses this IP to communicate with the other host which is outside of LTE $n / w$ i.e. in external n/w.

- Home Subscription Server (HSS): It is basically a repository for all the information related to user profile and different subscription information. It also stores the location of UE when user is in under control of $n / w$ other than home $n / w$.

\section{Protocol Stack \& BeARER}

Likewise, every communication protocol stack, LTE architecture also has two protocols stack i.e. Control plane and User plane as shown in Fig. 3 and Fig. 4 respectively. Fig. 3 and Fig. 4 depict the various protocol layers at different interfaces of control/user plane stack.

- Control Plane Stack (C-Plane): It basically provide assistance for the delivery for user traffic ex- link monitoring, mobility management, handover, radio resource allocation etc. During the ongoing traffic session, UE monitor the DL reception quality of the channel and send the Measurement Report (MR) towards the eNB. Based on these MR report eNB takes the decision for handover.

- User Plane Stack (U-Plane): It is basically responsible for the delivery of application layer data or simply says user traffic from one node to another node. It deals with efficient, error free and in sequence delivery of user traffic. User traffic mean data initiated from the Application (APP) layer, it may be http request, ftp request or voice call (speech frame generated from the Speech encoder). 


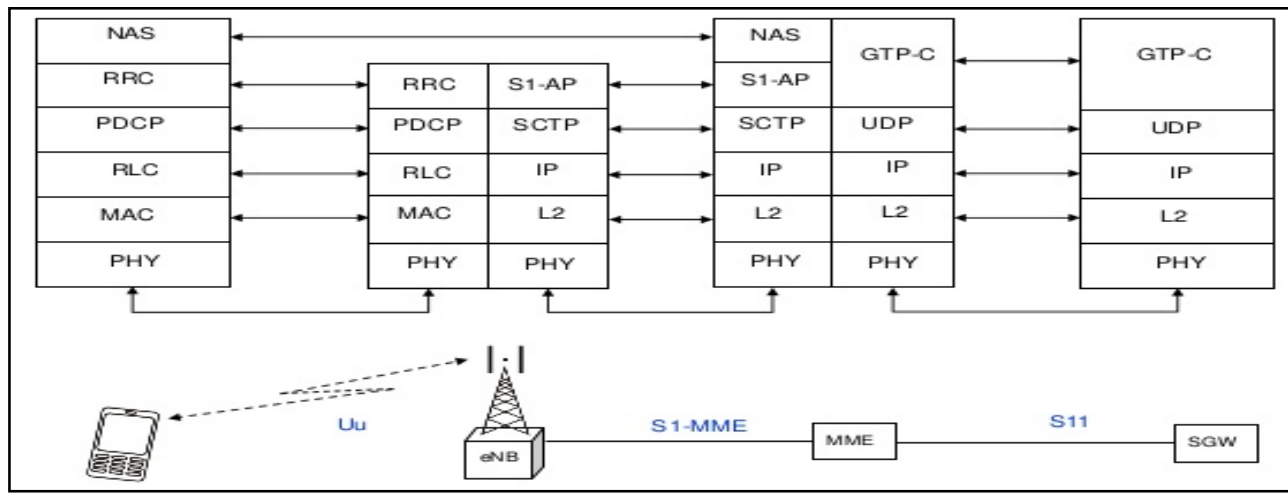

Fig.3. Control Plane Stack

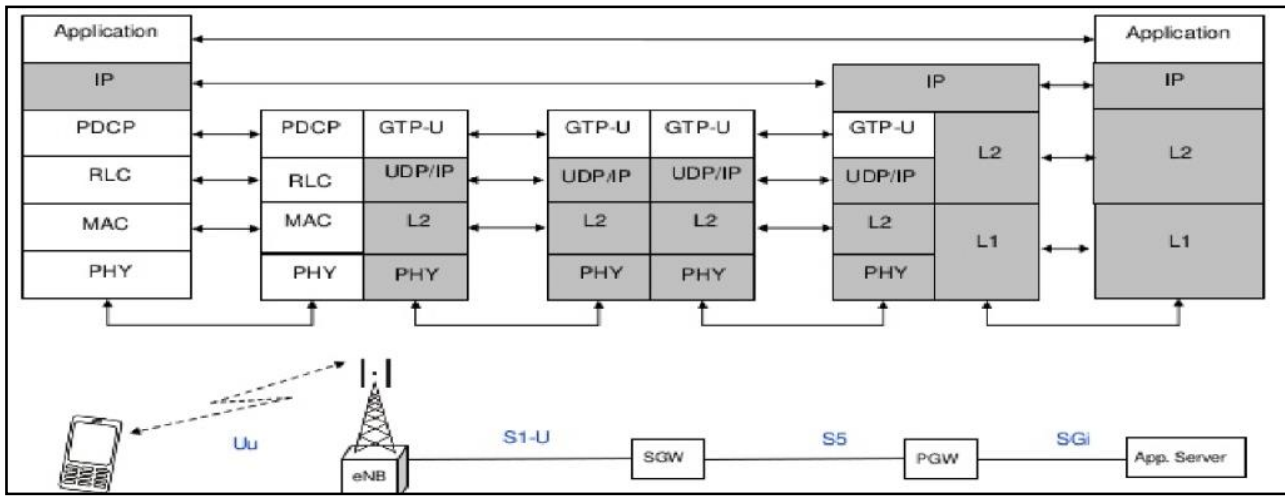

Fig.4. User Plane Stack

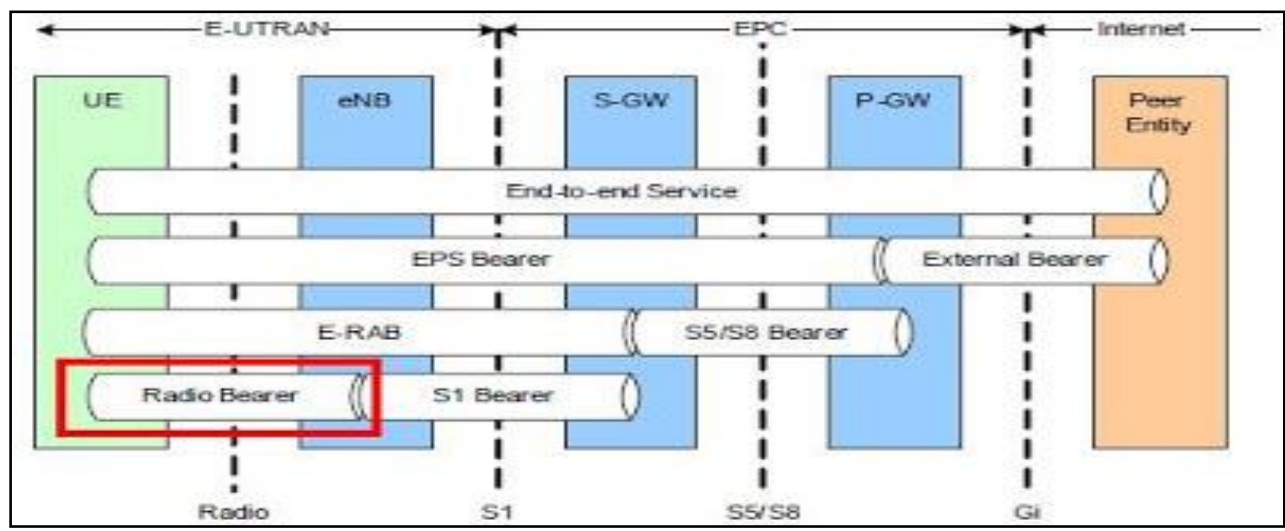

Fig.5. LTE Bearer

\section{A. Radio Protocol Stack $(U u)$}

It is the protocol stack which works on the air interface (Uu) [6] between the UE and eNB. Our study works mainly focus on this radio stack. The U-plane radio stack consists of four layers as shown in Fig. 4.

- Packet Data Convergence Protocol (PDCP): The major functions of this layer are header compression, integrity protection, ciphering and re-ordering during handover [7].

- Radio Link Control (RLC): The main functions of RLC layer are segmentation/re-segmentation, re-assembly, duplicate detection, re-ordering and ARQ re-transmission [8].

- Medium Access Control (MAC): The main functions of MAC layer are resource request
(Scheduling Request), Buffer Status Reporting (BSR), Logical Channel Prioritization (LCP), multiplexing/de-multiplexing and scheduling [9].

- PHY: The main functions of PHY layer are modulation \& demodulation, frequency and time synchronization, rate matching etc. [10].

\section{B. Bearer in LTE Architecture}

A bearer is a path through which traffic flow (User/Control plane) between different elements of the LTE $n / w$ as shown in Fig. 5. It is basically a virtual connection between two endpoints.

In our work, we will focus on the EPS bearer and Radio Bearer (RB).

EPS bearer exists between UE \& P-GW as shown in Fig.

5. There are two types of EPS bearer as given [12]: 
- Default EPS bearer: When UE attaches to the $\mathrm{n} / \mathrm{w}$ (say after power on), it will be assigned a default bearer which remains as long as UE is attached to the $n / w$. During the establishment of default bearer, UE gets an IP address allocated by P-GW. UE may have multiple default bearers but there exists only one default bearer corresponding to one Access Point Name (APN). Thus a LTE attached UE may have multiple Internet Protocol (IP) addresses each corresponds to one default bearer.

- Dedicated EPS bearer: It provide the specific tunnel to one or more specific traffic (i.e. VoIP, video etc.). Each dedicate bearer is associated with a default bearer which we called as Linked EPS bearer and multiple dedicated bearers can be associated with the same default bearer. Both EPS bearer (default and linked dedicated bearer) share the same IP address.

RB exists between UE \& eNB on the air interface (Uu). There are two types of Radio Bearer as follows:

- $\quad$ Signaling Radio Bearer (SRB): SRB are used to transfer the signaling/control plane information between the UE \& eNB. There exists three SRB i.e. SRB0, SRB1 and SRB2 These SRBs are configured during the RRC connection setup procedure [11].

- Data Radio Bearer (DRB): DRB are used to transfer the user plane traffic between UE \& eNB. DRB basically is a service provisioned at air interface. It facilitates the QoS at the air interface.

\section{Bearers Mapping with Logical Channels}

- $\quad$ There exists three SRB i.e. SRB0, SRB1 and SRB2 and corresponding logical channel identities are 0 , 1 and 2 respectively [9].

- There exists at max 8 DRB and corresponding logical channel identities are 3 to 10 respectively [9].

- Mapping between EPS bearer and DRB is one to one, thus there can exist at max 8 EPS bearer.

- $\quad$ EPS bearer ID = DRB ID + 4 and Logical channel $\mathrm{ID}=\mathrm{DRB}$ ID +2 .

\section{LOGICAL CHANNEL PRIORITIZATION (LCP) \& BUFFER STATUS REPORT (BSR)}

This section describes a brief overview of LCP \& buffer status report defined by the 3GPP standard [9]. It will describe the associated parameters and procedural description of LCP \& BSR.

\section{A. Logical Channel Prioritization Parameters}

eNB signal some parameter to the UE which are used during the uplink scheduling for each logical channel as follows -
- Priority: This defines the priority of each configured logical channel where a larger value indicates the lower priority level [9].

- Prioritized Bit Rate (PBR): This defines the average bit rate for each logical channel which UE should be able to fulfill to meet the provisioned QoS defined for this channel [9]. Unit of PBR is Kilobytes per second (KBps).

- Bucket Size Duration (BSD): This defines the upper time limit for continuous accumulation of data for each logical channel. This parameter is used to avoid starvation for lower priority logical channel [9]. Unit of BSD is in milliseconds (ms).

The range of parameter defined for each logical channel is given in Table 1.

Table 1. Logical Channel Parameters

\begin{tabular}{|c|c|}
\hline Priority & $1 \sim 16$ \\
\hline Prioritized Bit Rate (PBR) & $0 \mathrm{KBps} \sim 2048 \mathrm{KBps}$ \\
\hline Bucket Size Duration (BSD) & $50 \mathrm{~ms} \sim 1000 \mathrm{~ms}$ \\
\hline
\end{tabular}

\section{B. Logical Channel Prioritization Algorithm}

The UE contains a variable $\mathrm{B}_{\mathrm{L}}$ for every configured logical channel L. Variable $\mathrm{B}_{\mathrm{L}}$ is initialized to zero when the respective logical channel is configured. It is incremented by the PBR for each TTI (Transmission Time Interval) but the value of $\mathrm{B}_{\mathrm{L}}$ cannot exceed beyond the defined bucket size for the particular logical channel L. If the value of $\mathrm{B}_{\mathrm{L}}$ is greater than the defined bucket size, then it is set to defined bucket size only. The upper bound of bucket size for a logical channel $\mathrm{L}$ is equal to $\mathrm{PBR} \times \mathrm{BSD}$.

Fig. 6 shows the pictorial presentation of Logical Channel Prioritization. It is showing how the data is picked up from the different logical channel by considering the grant provided for the current TTI and configured priority of each channel.

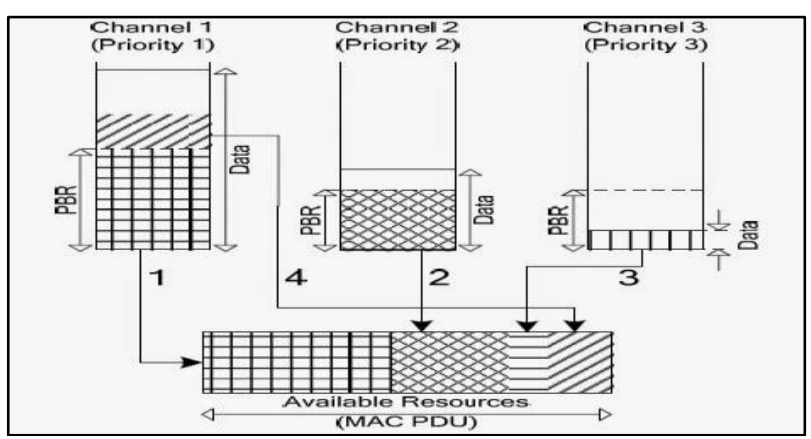

Fig.6. Logical Channel Prioritization

\section{Buffer Status Report (BSR)}

As in the LTE $\mathrm{n} / \mathrm{w}$, grant allocation is totally controlled by eNB, so whenever there is a new transmission of data it is necessary to notify the eNB about the amount of data waiting for transmission in the buffer so that eNB can allocate the necessary grant for the transmission of available data. BSR reporting is controlled by eNB with configuration of two timers as given below: 
- RetxBSR-Timer: When a BSR is included in the MAC PDU, this timer is started. It is restarted for every uplink grant received by UE provided that it is already running. If the timer expires and UE has the data available for transmission, then BSR is triggered. It is used to avoid the stall situation when UE think it has sent the BSR to eNB and actually eNB did not receive it. Possible range of timer value is $320 \mathrm{~ms} \sim 10240 \mathrm{~ms}$.

- PeriodicBSR-Timer: When a BSR is included in the MAC PDU, this timer is started and at the expiry of the timer a BSR is triggered again. Possible range of timer value is $5 \mathrm{~ms} \sim 2560 \mathrm{~ms}$.

When a BSR has been triggered and UE has uplink grant for this TTI then BSR is included in the MAC PDU otherwise a scheduling request shall be triggered [9].

\section{Scheduling Request (SR)}

The scheduling request is used by UE to request the uplink resources for the new transmission. At starting initialize the SR_COUNTER to 0. Below is the SR procedure [9] -

- If UE has no valid Packet Uplink Control Channel $(\mathrm{PUCCH})$ resources for SR request, then initiate a Random Access procedure (RACH).

- Else if UE has valid SR resources-

\section{○ If SR_COUNTER < dsr-TransMax}

- Increment SR_COUNTER by 1 .

- Send SR on PUCCH.

- Else initiate the RACH procedure.

Parameters configured by the $\mathrm{n} / \mathrm{w}$ which controls the scheduling request procedure as given below:

- dsr-TransMax: This defines the maximum number of times SR request can be sent before triggering the RACH procedure. Maximum value of this attribute is 64.

- SR Periodicity: After transmitting SR on PUCCH, if UE doesn't receive the uplink grant then it re-sends the SR based on the periodicity. Maximum value of periodicity is $80 \mathrm{~ms}$.

- SR-Prohibit Timer: The timer is used to prohibit the re-transmission of SR until it expires. Range of this is $0 \sim 7$ in multiple of SR periodicity. Value 0 means no timer is configured, value 1 means one SR period and so on.

\section{RELATED WORK}

In this section, we present the brief overview of available studies for the Logical Channel Prioritization. In [9], 3GPP defined a standard approach for LCP procedure. Author in [13] describe an analytical study \& performance evolutional approach for the MAC scheduler in the DL direction. Many configurable parameters have been considered which directly influences the performance scheduling and resultant effect on the provisioned QoS. In [14], author proposed a QoS scheduling approach. Resources are being allocated to the UE as per the QoS of the Radio Bearers. The proposed scheduler guarantees provisioned QoS and achieve an acceptable performance in terms of system performance. It also shows that when a particular UE suffers poor channel conditions, the scheduler still ensures the fairness to the UE with the minor reduction in overall system performance. [15] Another QoS aware scheduling algorithm has been proposed which is based on Service Type. Authors have proposed the algorithm considering multiple services having different provisioned QoS in LTE system. It combines the QoS with the user channel quality indication. In [16], author defined an approach to maximize the video quality. It is shown that one can obtain the gain in video quality by determining the QoS on per stream basis rather than user based approach. In [17], author describes the uplink and downlink physical channel structure and properties. The physical channel properties directly or indirectly influence the cell throughput resultant effects on the QoS. Mainly all the mentioned approach under this section is defined for the downlink direction and which briefly aims to achieve the QoS and overall system throughput based on the different parameters. No much related work has been done for the Uplink scheduling which is equal important as downlink scheduling. Our main contribution is to propose a QoS aware logical channel prioritization for UL direction. Our algorithm considers every data stream in order of the logical channel priority and which aims to achieve the PBR on average for each logical channel based on the decreasing priority order.

\section{PROPOSED IMPROVEMENTS}

In this section, we present improvements to the Logical Channel Prioritization in uplink to meet the provisioned QoS. This allows us to find the research direction that will influence the provisioned QoS for the different data stream. In the standard 3GPP approach, the bucket size for each configured logical channel is equal to the product of PBR * BSD except for the logical channel whose PBR is infinity. Under our proposed algorithm, we do not restrict the bucket size value to product of (PBR * BSD) instead we keep increasing size of bucket by PBR amount in every TTI. The standard defined algorithm does not meet the defined QoS for the logical channel under some certain conditions as follows:

- If UE is in a condition where eNB is allocating low UL resource grant (i.e. UE is sending less than PBR amount of data in each TTI for a certain time continuously) for Packet Uplink Shared Channel (PUSCH) and there is continuously incoming data from higher layer, then under such situation after a certain time bucket size will be full. Once the 
bucket size will be full then standard approach does not consider any more data from higher layer in subsequent TTI and resultant provisioned QoS does not fulfill.

- Under certain conditions, existing system will fail to meet the provisioned QoS when RetxBSR timer expires and there is no UL grant.

- Existing system does not fulfill the defined QoS when SR request failed even after maximum number of retry (dsr-TransMax).

- Under certain conditions, existing system does not able to fulfill the provisioned QoS when PeriodicBSR timer is configured with Max value. To trigger the BSR report, UE wait for the expiry of PeriodicBSR timer. In case, when it is configured with Max value then existing system buffer size will overflow before expiry of this timer provided that data is coming continuously from APP layer.

In our approach we keep accumulating the incoming data from higher layer even though bucket size reaches to product of (PBR * BSD). Thus UE can transmit big chunk of data once it gets the opportunity of larger uplink grant. Thus our approach will provide more flexibility to achieve the QoS under dynamic nature of grant allocation. In our study for accumulation of data, we assume that MAC layer buffer size is very big or infinity. Thus MAC layer buffer capacity directly impacts the flexibility of our algorithm.

\section{A. Effect of Bucket size over the provisioned QoS}

This section describes how bucket size limitation will affect the provisioned QoS corresponding to a logical channel. Limitation of buffer size will reduce the flexibility of UL data scheduling. Table $2 \& 3$ shows the bucket size corresponding to each possible value of BSD for PBR rate $8 \mathrm{KBps} \& 2048 \mathrm{KBps}$ respectively. It shows how many maximum data bytes can be accumulated in the buffer with different value of BSD. It also shows how many bytes are accumulated per TTI. Once the bucket size gets full then no more data will be accumulated further for a logical channel.

Table 2. Bucket size for $8 \mathrm{KBps}$ PBR Rate

\begin{tabular}{|c|c|c|}
\hline BSD $(\mathrm{ms})$ & Data per TTI (bytes) & Bucket Size (bytes) \\
\hline 50 & 8 & 400 \\
\hline 100 & 8 & 800 \\
\hline 150 & 8 & 1200 \\
\hline 300 & 8 & 2400 \\
\hline 500 & 8 & 4000 \\
\hline 1000 & 8 & 8000 \\
\hline
\end{tabular}

Table 3. Bucket Size for 2048 KBps PBR Rate

\begin{tabular}{|c|c|c|}
\hline BSD $(\mathrm{ms})$ & Data per TTI (bytes) & Bucket Size (bytes) \\
\hline 50 & 2048 & 102400 \\
\hline 100 & 2048 & 204800 \\
\hline 150 & 2048 & 307200 \\
\hline 300 & 2048 & 614400 \\
\hline 500 & 2048 & 1024000 \\
\hline 1000 & 2048 & 2048000 \\
\hline
\end{tabular}

Table 4. Min \& Max bucket size for each PBR

\begin{tabular}{|c|c|c|c|}
\hline $\begin{array}{c}\text { PBR } \\
(\mathrm{KBps})\end{array}$ & $\begin{array}{c}\text { Data per TTI } \\
\text { (bytes) }\end{array}$ & $\begin{array}{c}\text { Min Bucket } \\
\text { size (bytes) }\end{array}$ & $\begin{array}{c}\text { Max Bucket } \\
\text { Size (bytes) }\end{array}$ \\
\hline 8 & 8 & 400 & 8000 \\
\hline 16 & 16 & 800 & 16000 \\
\hline 32 & 32 & 1600 & 32000 \\
\hline 64 & 64 & 3200 & 64000 \\
\hline 128 & 128 & 6400 & 128000 \\
\hline 256 & 256 & 12800 & 256000 \\
\hline 512 & 512 & 25600 & 512000 \\
\hline 1024 & 1024 & 51200 & 1024000 \\
\hline 2048 & 2048 & 102400 & 2048000 \\
\hline
\end{tabular}

Table 4 shows the data bytes accumulated per TTI, minimum and maximum bucket size corresponding to each PBR rate.

Fig. 7 shows the pictorial representation of data accumulation corresponding to each BSD value for different PBR rate. By referring the Fig. 7 \& Table 4, consider 32 KBps PBR rate and Bucket size 1600 bytes. So if under some certain conditions, bucket size reaches value of 1600 bytes (bucket size full) and still there is no UL grant from the $\mathrm{n} / \mathrm{w}$ side, under this condition all incoming data from higher layer will not be accumulated further and thus it cause lacking the fulfillment of provisioned QoS for that particular logical channel.

\section{Bucket Size vs BSD}

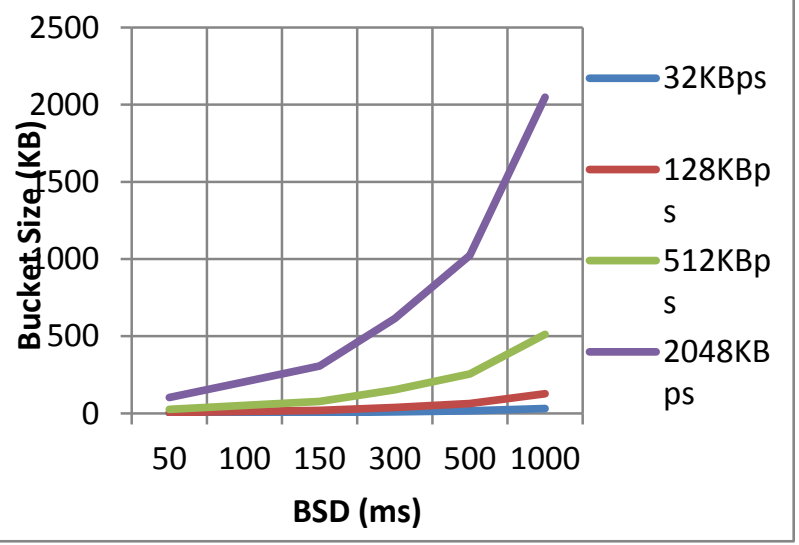

Fig.7. Bucket Size Vs BSD corresponding to PBR rate

\section{B. Impact of RetxBSR Timer on Bucket size}

Table 5. Data Accumulation in Min RetxBSR timer vs. Min Bucket Size

\begin{tabular}{|c|c|c|}
\hline $\begin{array}{c}\text { PBR } \\
(\mathrm{KBps})\end{array}$ & $\begin{array}{c}\text { Data accumulation in Min } \\
\text { RetxBSR Time (bytes) }\end{array}$ & $\begin{array}{c}\text { Min Bucket Size } \\
\text { (bytes) }\end{array}$ \\
\hline 8 & 2560 & 400 \\
\hline 16 & 5120 & 800 \\
\hline 32 & 10240 & 1600 \\
\hline 64 & 20480 & 3200 \\
\hline 128 & 40960 & 6400 \\
\hline 256 & 81920 & 12800 \\
\hline 512 & 163840 & 25600 \\
\hline 1024 & 327680 & 51200 \\
\hline 2048 & 655360 & 102400 \\
\hline
\end{tabular}


RetxBSR timer is getting start when a MAC PDU containing a BSR is transmitted. Timer range is 320ms $10240 \mathrm{~ms}$. Table 5 shows how much data would be accumulated in minimum value of RetxBSR timer (320 $\mathrm{ms}$ ). It also shows the minimum configurable bucket size corresponding to each PBR rate. This table shows that minimum bucket size is full before expire of RetxBSR timer.

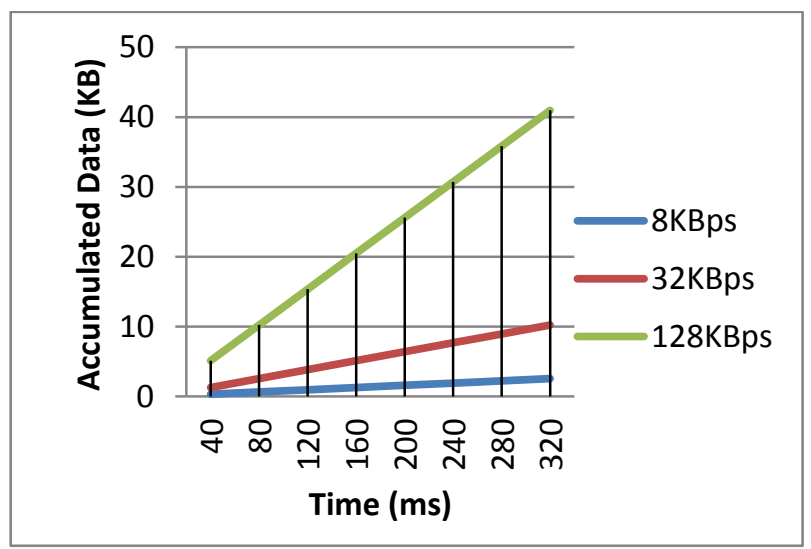

Fig.8. Data accumulated during running of RetxBSR timer

In Table $5 \&$ Fig. 8 , we have shown the data accumulation during the running of RetxBSR timer. In Fig. 9, we have shown that RetxBSR timer is started when BSR is triggered [9].

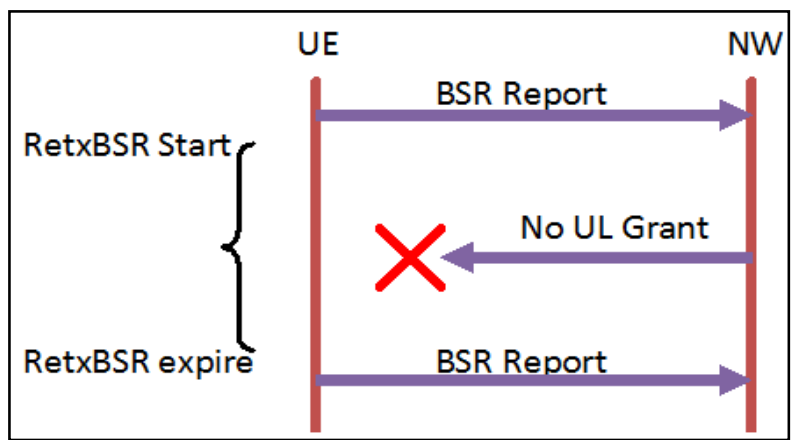

Fig.9. No UL Grant at expiry of RetxBSR

By referring the Table 5, Fig. $8 \& 9$, we can conclude that if there is no UL grant during running of RetxBSR timer then existing system bucket size will be full and further data will not be accumulated resultant provisioned QoS does not fulfill. In our modified approach, we keep accumulating the data during this time and thus UE has the flexibility to transmit it later when it has the opportunity of large UL resource grant. Thus our approach is suitable to meet the provisioned QoS under the burst resource allocation conditions.

\section{CONCLUSION}

This paper has presented an overview of LTE Radio stack and Logical Channel Prioritization in uplink. The proposed algorithm is effective under the burst resource allocation conditions with assumption of large buffer size for layer 2 (MAC). One important characteristic of this effort is that the new protocol builds upon the old one rather than proposing the new solutions, thus this can be adopted easily in the existing system. Another important characteristic is that our proposed methodology fulfills the QoS of application data according to the priority of data under burst resource allocation conditions.

\section{REFERENCES}

[1] Brahima Sanou. ICT Facts and Figures. ITU, 2016.

[2] TS 21.101. Universal mobile telecommunications system (UMTS); technical specifications and technical reports for a UTRAN-based 3GPP system. 3GPP, 2009.

[3] Fahimeh Rezaei, Michael Hempel, Hamid Sharif. LTE PHY Performance Analysis under 3GPP Standards Parameters. IEEE 2016.

[4] 3GPP TS 36.401. E-UTRAN architecture description (Release 8).

[5] Dr. Harri Holma. LTE for UMTS Evolution to LTE-Advanced Second Edition.

[6] 3GPP TS 36.300. E-UTRA \& E-UTRAN overall description (Release 8).

[7] 3GPP TS 36.323. Technical specification group Radio Access Network; E-UTRA; Packet Data Convergence Protocol (PDCP) specification (Release 8).

[8] 3GPP TS 36.322. E-UTRA; Radio Link Control (RLC) protocol specification (Release 10).

[9] 3GPP TS 36.321. E-UTRA; Medium Access Control (MAC) protocol specification (Release 8).

[10] 3GPP TS 36.201. E-UTRA; Long Term Evolution (LTE) physical layer; General description (Release 8).

[11] 3GPP TS 36.331. E-UTRA; Long Term Evolution (LTE) Radio Resource Control (RRC); General description (Release 8).

[12] 3GPP TS 24.301. E-UTRA; Technical Specification Group Core Network and Terminals; Non-Access-Stratum (NAS) protocol for Evolved Packet System (EPS).

[13] Naila Bouchemal, Nora Izri, Samir Tohme. MAC-LTE Scheduler Modeling and Performance Evaluation in LTE Network. PIMRC, 2014 IEEE 25th Annual International.

[14] Badri Nayak, A. M. Prasad, Mohamed Niaz. LTE MAC Scheduler Analysis based on QoS Aware Proportional Fair Scheduling Algorithm. IJIRCCE August, 2015.

[15] Weihong Fu, Qingliang Kong, Weixin Tian, Cheng Wang, Lili Ma. A QoS-Aware Scheduling Algorithm Based on Service Type for LTE Downlink. ICCSEE, 2013.

[16] A. Balasubramanian, A. Rapaport, W.Liu. VIDEO AWARE LOGICAL CHANNEL BASED SCHEDULING FOR LTE SYSTEMS. InterDigital Communication.

[17] A. Z. Yonis, M. F. L. Abdullah, "Downlink and Uplink Physical Channels in Long Term Evolution", International Journal of Information Technology and Computer Science (IJITCS), vol.4, no.11, pp.1-10, 2012. DOI: 10.5815/ijitcs.2012.11.01. 
[18] Goudru N.G, Vijaya Kumar B.P, "Performance Analysis of TCP and its Enhancement for Quality of Service in Mobile Wireless Networks in Single Traffic Using Prioritised Hard Handoff (PH2)", International Journal of Information Technology and Computer Science (IJITCS), Vol.8, No.5, pp.88-94, 2016. DOI:10.5815/ijitcs.2016.05.10.

\section{Author's Profiles}

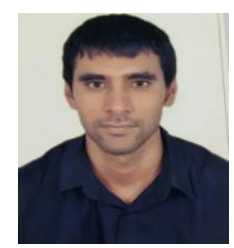

Mannu Kumar, currently working in the mobile division of Samsung Electronics Pvt. Ltd, where he is involved in the research and development of various smart phones which supports LTE technology. He has worked over more than 100 smart phones launched by Samsung in the different regions of the world. He has received his B. Tech degree in Computer Science from MMU Mullana, with honors in 2010. Currently he is pursuing his part time M. Tech degree from NCU, Gurgaon.

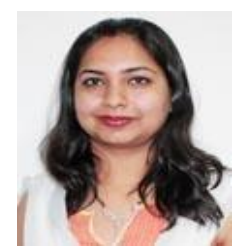

Neeti Kashyap, is pursuing her $\mathrm{PhD}$ degree in the field of Internet of Things (IoT). She has received her B. Tech and M. Tech degree from GGS Indraprastha University, Delhi. Currently she is working as an Assistant Professor in the dept. of CSE \& IT at NCU Gurgaon, where she is involved in research and teaching of UG and PG students. She has published more than 10 research papers in International/National Conferences and International Journals.

How to cite this paper: Mannu Kumar, Neeti Kashyap,"QoS Aware Logical Channel Prioritization under Burst Resource Allocation for Uplink in LTE", International Journal of Information Technology and Computer Science(IJITCS), Vol.9, No.8, pp.14-21, 2017. DOI: 10.5815/ijitcs.2017.08.02 